\title{
Warning About Bacterial Contamination of Therapeutic Contact Lenses
}

\author{
D. P. O'NEILL and M. M. MATHESON
}

London

\begin{abstract}
Summary
Three of twenty-six soft therapeutic contact lenses were found to have bacterial contaminants in their original sealed manufacturers' containers. We wish to alert clinicians to this danger.
\end{abstract}

\begin{abstract}
Methods
Twenty-six soft, therapeutic, bandage contact lenses were randomly selected from stock in Moorfields Eye Hospital. The lenses came from a total of eight batches and had been produced by four different manufacturers. All lenses were tested before their expiry date where such a date was marked on their container (only two out of four manufacturers had marked their lens containers with an expiry date). Permalens and Moorfields Eye Hospital high water content lenses were selected as well as Bausch and Lomb and Hoya low water content lenses. The four manufacturers confirmed that their normal decontamination procedure involved autoclaving lenses sealed in containers of unpreserved saline.

The lenses were inspected in their containers and any opacities of the lens or surrounding fluid noted. None of the lenses had been re-packaged or re-sterilised. The original sealed manufacturer's containers were opened. The contact lenses and the fluid surrounding the contact lenses were cultured. The fluid and lenses were cultured on blood agar, thioglycollate, Robertson's cooked meat broth and Sabouraud's media using conventional aseptic techniques. The culture plates were incubated microaerophilically (in
\end{abstract}

$10 \%$ carbon dioxide) at $37^{\circ} \mathrm{C}$. The fungal cultures were incubated at $37^{\circ} \mathrm{C}$ for three days. The fungal plates were then cultured at room temperature for 28 days.

\section{Results}

Naked eye inspection showed that only one of the twenty-six lens containers had turbidity of the fluid surrounding the lens. The remainder of the containers, fluid and contact lenses appeared normal to the naked eye.

Three specimens $(11.5 \%)$ were found to be contaminated. The turbid fluid was found to be contaminated with Escherica coli. Two further containers were found to be contaminated with Staphylococcus epidermidis. The remaining twenty three $(88.5 \%)$ specimens were sterile on culture.

No fungal growth was detected despite culture for four weeks on Sabouraud's medium. No viral culture or acanthomoebae isolation techniques were used.

\section{Discussion}

Some eyes are more prone to bacterial keratitis than others. An eye that requires a therapeutic contact lens often has a condition which predisposes the patient to secondary bacterial keratitis prior to the insertion of a contact lens. These conditions include bullous 
corneal endothelial decompensation, recurrent corneal erosions, corneal perforation and corneal wound dehiscence. ${ }^{1,2}$ Contact lenses are risk factors for the development of microbial keratitis in otherwise normal eyes. This increased risk can be demonstratęd in subjects who wear cosmetic contact lenses. ${ }^{3,4,5}$ The incidence of contact lens related microbial keratitis is particularly marked in those using extended wear cosmetic soft contact lenses. ${ }^{6,7}$

The insertion of an extended wear soft contact lens into an eye which is already vulnerable to keratitis for other reasons is associated with a definite risk of developing microbial keratitis. ${ }^{8}$

It is difficult to draw conclusions about the role of soft lenses in these cases as there is no suitable control population with which to compare the results.

It is clear that microbial contamination of ophthalmic preparations at the time of manufacture can result in serious ocular morbidity. ${ }^{9}$ It is important to note that the insertion of microbially contaminated material onto the eye does not necessarily result in infective complications. $^{10}$

Effective disinfection and sterilisation of soft contact lenses has been a problem since their introduction in the early 1960s. Ruben noted the presence of fungus in the Wichterle's new Czeck polymers as early as $1966 .{ }^{11}$ One of the problems with soft contact lenses is that they can harbour pathogens which are able to adhere to their surface. ${ }^{12}$

The commonest microbial decontamination methods use heat or chemical agents. Both of these methods have their problems when applied to soft contact lenses. Steam autoclaving at $130^{\circ} \mathrm{C}$ for ten minutes is considered sufficient to kill all bacterial and fungal elements on contact lenses. Unfortunately, soft lenses which contain polyvinyl pyrrolidone (PVP) or have a hydration of greater than $40 \%$ are subject to critical polymer degradation by repeated autoclaving. ${ }^{13}$ Pasteurisation is a useful non toxic disinfectant method which is used extensively by patients. It does not cause severe degradation of the polymers in soft contact lenses but the temperatures used are insufficient to kill all spores which may be present on contact lenses. ${ }^{13}$
Effective chemical contact lenses decontamination is hampered by problems with the quality, toxicity and efficacy of the agents used. This was demonstrated by the rejection and withdrawal of $47 \%$ of lens cleaners and other contact lens solutions when mandatory product licenses were introduced for these products in the United Kingdom. ${ }^{14}$ Many products which survived the scrutiny of the licencing body still had significant problems with toxicity. ${ }^{15}$

Preliminary results of ultraviolet light sterilisation of soft lenses are encouraging. ${ }^{16}$

Our study showed contamination of a substantial proportion $(11.5 \%)$ of soft bandage lenses intended for extended use in abnormal eyes despite apparently having been autoclaved. This represents an avoidable hazard for these compromised eyes. We note that one of the contaminated lenses was suspended in a cloudy solution. We suggest that the clinician always inspects contact lenses in their containers prior to dispensing.

\section{Conclusion}

We found an $11.5 \%$ incidence of microbial contamination of contact lenses in our series. We suggest that:

(1) Soft bandage lenses should be supplied with a clearly marked expiry date and with printed details on the storage fluid and method of microbial decontamination.

(2) Lenses and their containers should be carefully inspected prior to dispensing.

(3) If bandage lenses are inserted into eyes that are at risk of developing microbial keratitis it may be prudent to culture the storage medium for known pathogens.

We conclude that:

(1) Some soft contact lenses cannot be assumed to be sterile even in their original manufacturer's sealed package.

(2) The efficacy of soft contact lens microbial decontamination requires further investigation.

\section{References}

${ }^{1}$ Ruben M and Khoo CY: Therapeutic contact lenses in Contact lenses. PG Publishing, Singapore. 1989: 59.

${ }^{2}$ McDermott ML and Chandler JW: Therapeutic uses of contact lenses. Surv Ophthalmol 1989, 33: 381-94.

${ }^{3}$ Liesegang TL: Bacterial and fungal keratitis. In 
Kaufmann HE, Barron BA, McDonald MB, Waltman SR eds. The Cornea: Churchill Livingstone 1988: 217-70.

${ }^{4}$ Galentine PG, Cohen EJ, Laibson PR, Adams C, Michaud R, Arentsen JJ: Corneal ulcers associated with contact lens wear. Arch Ophthalmol 1984, 102: 891-4.

${ }^{5}$ Dart JK: Predisposing factors in microbial keratitis: the significance of contact lens wear. $\mathrm{Br} J \mathrm{Oph}$ thalmol 1988, 72: 926-30.

${ }^{6}$ Bailey CS: A review of relative risks associated with four types of contact lenses. Cornea 1990, 9 (suppl 1): pp 59-61.

${ }^{7}$ Schein OD and Poggio EC: Ulcerative keratitis in contact lens wearers. Cornea 1990,9 (suppl 1): pp 55-58.

${ }^{8}$ Brown SI, Bloomfield S, Pearce DB, Tragakis M: Infections with the therapeutic soft lens. Arch Ophthalmol 1974, 91: 275-7.

${ }^{9}$ Eye Hospital Report. Pharm J 1965, 195: 526.
11) Pitts RE and Krachmer JH: Evaluation of soft contact lens disinfection in the home environment. Arch Ophthalmol 1979, 97: 470-2.

${ }^{11}$ Ruben M: Preliminary observations of soft (hydrophilic) contact lenses. Proc R Soc Med 1966, 59: 531-2.

12 Aswad MI, John T, Barza M, Kenyon K, Baum J: Bacterial adherence to soft contact lenses. Ophthalmology 1990, 97: 296-302.

${ }^{13}$ Morgan JE: Soft contact lens hygiene-Clinical experience in Dabezies $\mathrm{OH}$, Contact lenses, 2nd ed. Little Brown, Boston. 1988: 40 A3.

${ }^{14}$ Meakin BJ: Contact lens solutions in the United Kingdom. J B Cont Lens Assoc 1984, 7: 192-203.

${ }^{15}$ Morgan JF: Complications associated with contact lens solutions. Ophthalmology 1979, 86: 1107-19.

${ }^{16}$ Dolman PJ and Dobrogowski MJ: Contact lens sterilisation by ultraviolet light. Am J Ophthalmol 1989, 108: 665-9. 